\title{
Pneumocystis pneumonia in children - the relevance of chemoprophylaxis in different groups of immunocompromised and immunocompetent paediatric patients
}

\author{
OLGA ZAJAC-SPYCHAEA', EWELINA GOWIN², PIOTR FICHNA ${ }^{3}$, JACEK WYSOCKI ${ }^{4}$, \\ MARTA FICHNA ${ }^{5}$, ARLETA KOWALA-PIASKOWSKA ${ }^{5}$, IWONA MOZER-LISEWSKA ${ }^{6}$, \\ JERZY NOWAK ${ }^{5}$, DANUTA JANUSZKIEWICZ-LEWANDOWSKA ${ }^{1,5,7}$
}

\author{
${ }^{1}$ Department of Oncology, Hematology and Bone Marrow Transplantation, Poznan University of Medical Sciences, Poznan, Poland \\ ${ }^{2}$ Family Medicine Department, Poznan University of Medical Sciences, Poznan, Poland \\ ${ }^{3}$ Department of Diabetology, Poznan University of Medical Sciences, Poznan, Poland \\ ${ }^{4}$ Department of Health Promotion, Poznan University of Medical Sciences, Poznan, Poland \\ ${ }^{5}$ Department of Molecular Pathology, Institute of Human Genetics, Polish Academy of Sciences, Poznan, Poland \\ ${ }^{6}$ Department of Infectious Diseases, Poznan University of Medical Sciences, Poznan, Poland \\ ${ }^{7}$ Department of Medical Diagnostics, Poznan University of Medical Sciences, Poznan, Poland
}

\begin{abstract}
Introduction: Pneumocystis jirovecii is an opportunistic pathogen causing pneumocystis pneumonia $(P C P)$, a life-threatening infection, in immunocompromised patients. In this study, retrospective analysis of the presence of $P$. jirovecii DNA in different samples collected from children with suspected PCP was carried out.

Material and methods: Three hundred and six specimens [152 bronchoalveolar lavage (BAL) specimens, 80 blood specimens, 18 bronchial secretions (BS), 34 induced sputum samples, 10 endotracheal aspirates (ETA), and 12 other type samples] obtained from patients with suspected $P C P$ were examined by real-time $P C R$.

Results: Forty (13.1\%) patients were positive for P. jirovecii: 4 (7.7\%) patients with malignancies, $3(6.8 \%)$ transplant recipients, 15 (23.1\%) other immunocompromised patients, and $18(12.4 \%)$ immunocompetent patients. Pneumocystis jirovecii DNA was detected in $20.4 \%$ of BAL specimens, $11.1 \%$ of BS samples, $10 \%$ of ETA sample, $8.8 \%$ of induced sputum samples, and in $3.7 \%$ of blood samples. Comparing the frequency of the presence of $P$. jirovecii DNA between the group of children treated with $P C P$ chemoprophylaxis (malignancy patients and transplant recipients) and a group of children not receiving this prophylaxis (other immunocompromised and immunocompetent children), we found that the occurrence of PCP was twice as high in the latter group of children (7.3\% and $15.7 \%$, respectively).

Conclusions: Respiratory samples, such as BS, BAL, or ETA specimens, are the material of choice for the diagnosis of PCP. Due to high incidence of PCP in certain groups of immunocompetent and immunocompromised patients, besides cancer patients and transplant recipients, consideration of $P C P$ prophylaxis is required in these groups as well.
\end{abstract}

Key words: pneumonia, pneumocystis, immunosuppression.

(Centr Eur J Immunol 2015; 40 (1): 91-95)

\section{Introduction}

Pneumocystis jirovecii (formerly known as Pneumocystis carinii) is an opportunistic pathogen causing pneumocystis pneumonia (PCP), a life-threatening infection, in immunocompromised patients [1]. Pneumocystis pneumonia is still the most common AIDS-defining ill- ness in developed countries; however, it may also occur in HIV-negative immunocompromised patients, including malignancy patients, transplant recipients, and patients receiving immunosuppressive therapy for autoimmune or inflammatory diseases [2]. In HIV-negative immunocompromised patients, the course of PCP is typically more

Correspondence: Ewelina Gowin, Poznan University of Medical Sciences, Przybyszewskiego 49, 60-355 Poznan, Poland, e-mail: ewego@poczta.onet.pl 
acute and severe than in HIV-positive patients. For this reason, the mortality rate in HIV-negative patients is higher and amounts to as much as about $35-55 \%$, compared to about $10-20 \%$ in HIV-positive patients [3]. The fatality rate in immunocompromised children with untreated PCP is approximately $100 \%$ [4].

Clinically, PCP is characterised by non-productive cough, shortness of breath, and profound hypoxaemia, lower than that expected from clinical manifestations. As $P$. jirovecii cannot be cultured from clinical specimens, diagnosis of PCP is typically made by direct examination of respiratory samples after conventional cytochemical staining (i.e. with toluidine blue or Giemsa and Wright stains) in order to detect the cyst form of $P$. jirovecii [5]. Sensitivity of the staining techniques ranges from $35 \%$ to $70 \%$ for bronchoalveolar lavage (BAL) and is much lower for specimens with low loads of $P$. jirovecii, such as in the case of immunocompromised, HIV-negative patients, in contrast to specimens derived from HIV-positive patients, which contain high loads of microorganisms [6]. Due to the low sensitivity of microscopic methods in HIV-negative patients, polymerase chain reaction (PCR)-based methods have become increasingly common for PCP diagnosis [7]. The PCR method can detect very low levels of $P$. jirovecii DNA; it is suggested that the molecular test is about 100 times more sensitive than microscopy [8]. Because of the high sensitivity and possibility of false positive results in conventional PCR, real-time PCR was introduced, allowing for quantification of $P$. jirovecii DNA load and application of cutoff values. This approach is intended to facilitate differentiation between colonization and infection [9]. Nevertheless, no clear answer as to how to distinguish these two situations by PCR methods has been given yet [10]. The PCR assay, widely used in diagnosing PCP from $\mathrm{BAL}$ or lung samples, is also a promising technology for respiratory samples obtained by non-invasive methods such as induced sputum, nasopharyngeal aspirate, or oral washing specimens $[1,8]$. However, sputum induction is not widely performed in children, because it may result in clinical deterioration or nosocomial transmission of respiratory pathogens. Therefore, non-invasive methods of PCP diagnosis are desirable, especially in paediatric patients [4]. Despite its frequent clinical usage, the diagnostic value of the PCR assay has not yet been evaluated for different types of clinical specimens.

In this study, retrospective analysis of the presence of $P$. jirovecii DNA in different samples collected from children with suspected PCP was carried out.

\section{Material and methods}

Between May 2006 and December 2012, 1156 specimens including bronchial secretions (BS), BAL specimens, induced sputum samples, endotracheal aspirates (ETAs), pharyngeal swab, vomit samples, and blood specimens were analysed by PCR for the presence of $P$. jirovecii DNA in the Department of Medical Diagnostics (Poznan University of Medical Sciences, Poland). Details are presented in Table 1.

Samples for molecular testing were obtained from adult and pediatric patients suspected for PCP, treated in different hospitals. Only one sample was taken from each patient. At the moment of sampling, all patients presented pulmonary symptoms such as cough, dyspnoea, and fever, accompanied by abnormal chest radiograms or computed tomography scans (interstitial pneumonia or ground glass opacities). The study group comprised 306 patients treated in the Children's University Hospital in Poznan. Samples for molecular testing were collected as part of routine diagnostic procedures and the type of specimen depended on the clinical status of the child and the access to invasive

Table 1. Type and percentage of positive results in samples examined for Pneumocystis jirovecii DNA in the Department of Medical Diagnostics (Poznan University of Medical Sciences, Poland)

\begin{tabular}{|c|c|c|c|c|c|c|c|}
\hline & All samples & Positive (\%) & Study group & Positive (\%) & Rest of the samples & Positive (\%) & $p$ value \\
\hline BAL & 559 & $68(12.2 \%)$ & 152 & $31(20.4 \%)$ & 407 & $37(6.8 \%)$ & 0.007 \\
\hline Blood & 284 & $8(2.8 \%)$ & 80 & $3(3.7 \%)$ & 204 & $5(2.4 \%)$ & $0.6909 \mathrm{NS}$ \\
\hline BS & 182 & $22(12.1 \%)$ & 18 & $2(11.1 \%)$ & 164 & $20(12.2 \%)$ & $1.0 \mathrm{NS}$ \\
\hline ETA & 39 & $2(5.1 \%)$ & 10 & $1(10 \%)$ & 29 & $1(3.4 \%)$ & $0.4521 \mathrm{NS}$ \\
\hline Sputum & 66 & $11(16.7 \%)$ & 34 & $3(8.8 \%)$ & 32 & $8(25 \%)$ & $0.1038 \mathrm{NS}$ \\
\hline URS & 20 & 0 & 10 & 0 & 10 & 0 & NA \\
\hline PF & 3 & 0 & 0 & 0 & 3 & 0 & NA \\
\hline Vomit & 2 & 0 & 2 & 0 & 0 & 0 & NA \\
\hline CSF & 1 & 0 & 0 & 0 & 1 & 0 & NA \\
\hline Total & 1156 & $111(9.6 \%)$ & 306 & $40(13 \%)$ & 850 & $71(8.3 \%)$ & 0.0231 \\
\hline
\end{tabular}

p value calculated for comparison of positive results in the study group with the rest of patients; BAL - bronchoalveolar lavage, BS - bronchial secretions, CSF - cerebrospinal fluid, ETA - endotracheal aspirates, PF - pleural fluid, URS - upper respiratory secretions, NA - not applicable 
diagnostic methods such as BAL or ETA. In patients with malignancies and hematopoietic stem cell transplant recipients, infections due to cytomegalovirus, Epstein-Barr virus (EBV), respiratory syncytial virus (RSV) and adenoviruses (ADV) were excluded by PCR testing. In other groups of patients, only CMV and RSV infections were excluded by determination of specific antibodies in the serum. Patients with malignancies and HSCT recipients had received PCP prophylaxis (sulfamethoxazole and trimethoprim $15 \mathrm{mg}$ / $\mathrm{kg}$ /day, three times a week) before sampling. No other children, either immunocompetent or other immunocompromised, received PCP chemoprophylaxis.

For real-time PCR, AmpliSens Pneumocystis jirovecii (carinii) FRT PCR kit (Ecoli s.r.o., Bratislava, Slovak Republic) was used. Results of $P$. jirovecii DNA amplification were recorded using the JOE/HEX fluorescence channel, and results of internal control DNA amplification were recorded using the FAM channel. Endogenous internal control facilitates verification of analytical PCR steps as well as assessment of sampling adequacy. Only a small number of samples (11) were screened for $P$. jirovecii using the conventional detection method, i.e. Giemsa stain. Due to the relatively low sensitivity of $P$. jirovecii detection by means of Giemsa staining in HIV-negative patients [6] and the small number of test samples in the analysed group of children, the usefulness of Giemsa stain and the molecular techniques was not compared in further studies.

\section{Results}

In the study group there were 152 girls and 154 boys, their mean age was 1.8 years, and range 2 months to 18 years. A total of 306 specimens, including $152 \mathrm{BAL}$ specimens, 80 blood specimens, 18 BS, 34 induced sputum samples, 10 ETAs, and 12 other-type specimens (10 pharyngeal swab and 2 vomit samples).

The study patients were divided into four groups, including: $52(17.0 \%)$ patients with malignancies (41 pa- tients with leukaemia and solid tumours (11 patients); $44(14.4 \%)$ transplant recipients (43 HSCT recipients and 1 liver transplant recipient); 65 (21.2\%) other immunosuppressed patients (48 patients with inherited or acquired immunodeficiency and 17 patients receiving immunosuppressive drugs for autoimmune diseases); and 145 (47.4\%) immunocompetent patients. The immunocompetent group consisted of paediatric patients with congenital heart (111 patients) or kidney diseases (11 patients), trauma patients staying in the intensive care unit (14), and patients with cystic fibrosis (9).

Forty out of 306 patients (13\%) were positive for $P$. jirovecii as determined by real-time PCR. Pneumocystis pneumonia was diagnosed in 4 out of $52(7.7 \%)$ patients with malignancies, 3 out of $44(6.8 \%)$ transplant recipients, 15 out of $65(23.1 \%)$ other immunocompromised patients, and in 18 out of $145(12.4 \%)$ immunocompetent patients.

Comparing the frequency of the presence of $P . j i$ rovecii DNA between the group of children treated with PCP chemoprophylaxis (malignancy patients and transplant recipients) and a group of children not receiving this prophylaxis (other immunocompromised and immunocompetent children), we found that the occurrence of PCP was twice as high in the latter group of children $(7.3 \%$ and $15.7 \%$, respectively) (Table 1 ).

Overall, $P$. jirovecii DNA was detected in 31 of 152 BAL samples (20.4\%), 2 of 18 BS (11.1\%), 1 of 10 ETAs (10\%), 3 of 34 sputum samples (8.8\%), and 3 of 80 blood samples $(3.7 \%)$. No $P$. jirovecii DNA was detected in pharyngeal swabs or vomit samples (Table 2 ).

\section{Discussion}

The study results revealed the highest detection rate of $P$. jirovecii DNA in respiratory tract samples (BS, BAL, ETA, or induced sputum $-92.5 \%$ of all positive samples). Only $7.5 \%$ of PCP diagnoses could be made on the basis

Table 2. Polymerase chain reaction-positive Pneumocystis jirovecii results according to the specimen type

\begin{tabular}{lccccc}
\hline Specimen type & $\begin{array}{c}\text { Malignancy } \\
\mathbf{n = 5 2}\end{array}$ & $\begin{array}{c}\text { Transplant recipients } \\
\boldsymbol{n}=\mathbf{4 4}\end{array}$ & $\begin{array}{c}\text { Immunocompromised** } \\
\boldsymbol{n = 6 5}\end{array}$ & $\begin{array}{c}\text { Immunocompetent*** } \\
\boldsymbol{n = 1 4 5}\end{array}$ & $\begin{array}{c}\text { Total positive } \\
\text { samples }\end{array}$ \\
\hline BAL & $2 / 8$ & $1 / 6$ & $14 / 37$ & $14 / 101$ & $31 / 152(20.4 \%)$ \\
\hline Blood & $2 / 26$ & $0 / 20$ & $0 / 18$ & $1 / 16$ & $3 / 80(3.7 \%)$ \\
\hline BS & $0 / 7$ & $1 / 5$ & $0 / 1$ & $1 / 5$ & $2 / 18(11.1 \%)$ \\
\hline ETA & $0 / 2$ & - & $1 / 1$ & $0 / 7$ & $1 / 10(10.0 \%)$ \\
\hline Sputum & $0 / 9$ & $1 / 7$ & $0 / 7$ & $2 / 11$ & $3 / 34(8.8 \%)$ \\
\hline Other*** & - & $0 / 6$ & $0 / 1$ & $0 / 5$ & $0 / 12$ \\
\hline Total positive samples & $4(7.7 \%)$ & $3(6.8 \%)$ & $15(23.1 \%)$ & $18(12.4 \%)$ & $40 / 306(13 \%)$ \\
\hline
\end{tabular}

$B S$ - bronchial secretions, BAL - bronchoalveolar lavage, ETA - endotracheal aspirates

*Patients with other acquired or inherited immunodeficiency and patients receiving immunosuppressant drugs for autoimmune diseases

**Patients with congenital heart or kidney diseases, trauma patients staying in the intensive care unit, and patients with cystic fibrosis

***Other-type samples included pharyngeal swabs and vomit samples 
of blood samples. Similar data regarding PCP diagnoses made from respiratory samples by means of PCR techniques were reported by Pinlaor et al. [11] and Samuel et al. [4]. Moreover, it has been demonstrated that $P$. jirovecii DNA detection in blood specimens is not a valuable method for diagnosing PCP $[12,13]$. Positive results obtained from blood samples as reported in the literature and observed in our study may be due to transient blood passages of Pneumocystis organisms, infections with highly virulent parasitic strains or the presence of residual parasite material in phagocytes [12].

As $P$. jirovecii cannot be cultured, no objective method is available to distinguish the disease from asymptomatic colonisation. In our study, all children were tested for PCP due to clinical manifestations of pneumonia, and those positive for $P$. jirovecii DNA were successfully treated for PCP. It seems surprising that over $19 \%$ of immunocompetent patients in the study had PCP as demonstrated by positive PCR findings. However, all these patients had underlying chronic diseases or severe injuries, which could be additional risk factors. In addition, most of these patients were in the first years of life (the mean age in the PCP-positive group of immunocompetent patients was 6.7 months), which is the period associated with the highest risk of PCP [14]. Initial infection normally occurs in childhood, and $94 \%$ of children have pneumocystis antibodies at the age of four years. Thus, it is highly improbable that positive PCR results in the above-mentioned group represents the disease rather than colonisation. The carriage of $P$. jirovecii has still not been reliably verified and the issue remains controversial. Therefore, people belonging to the group at high risk of opportunistic infections should be tested for $P$. jirovecii. Diagnosis should be done on the basis of comprehensive examination data including clinical examination, X-ray or CT scans, and laboratory analyses. Polymerase chain reaction is a very sensitive method that allows the detection of single copies of pathogen DNA. Consequently, this method should be used in the diagnostics of pneumocystis pneumonia as a supporting method, albeit not the main method.

The most important risk factor associated with PCP is immune dysfunction, mainly resulting from lymphocytopaenia related to the received treatment (high doses of corticosteroids, e.g. $>20 \mathrm{mg}$ of prednisolone daily for 4 weeks) [15]. Recently, new immunomodulatory agents, including anti-CD-52 (alemtuzumab) and anti-CD20 (rituximab) monoclonal antibodies as well as calcineurin inhibitors (sirolimus, tacrolimus) have been identified as other risk factors. Some of them impair the cellular immune response (alemtuzumab), whereas others impair the humoral immune response (rituximab). Thus, it seems that not only cellular immunity defects are responsible for $P$. jirovecii infection, and that $\mathrm{PCP}$ risk assessment in immunocompromised patients may be even more complicated [16].
Polymerase chain reaction prophylaxis is recommended in certain groups of immunocompromised patients, i.e. patients with malignancies (particularly haematological malignancies), transplant recipients, and HIV-positive patients with CD4+ cell counts lower than 200 cells/ $\mu$ l. The widespread usage of trimethoprim-sulfamethoxazole (TS) for PCP prophylaxis has resulted in the development of TS-resistant $P$. jirovecii strains. Huang et al. elucidated the drug resistance mechanism by sequencing the pneumocystis dihydropteroate synthase gene and correlating its mutations with clinical outcomes, including PCP treatment failure or death [13]. Although mortality in immunocompromised patients may be related to multiple factors other than PCP, further research on potential TS resistance is needed. Nevertheless, TS-resistant Pneumocystis should be considered to be the cause of PCP in patients receiving prophylaxis in our study.

Our study is retrospective, and it has several limitations. Only one sample was taken from each patient, so intra-sample variability could not be checked. Another limitation is that due to heterogeneity of samples, our study may lack the statistical power to determine which type of sample is best in diagnosing PCP. From 1156 samples, we selected a group of paediatric patients treated in the Children's University Hospital in Poznan, Poland. The rest of the samples were collected in different hospitals, and due to the heterogeneity of samples and underlying clinical conditions it would be difficult to draw conclusions. Comparing our study group to the rest of patients we detected a higher proportion of positive results. It might be caused by the younger age of our patients; there were only children in the study group.

\section{Conclusions}

Pneumocystis pneumonia in immunocompromised patients is a severe disease that may become life threatening if adequate treatment is not administered on time. It is well known that the fatality rate in children with untreated PCP is approximately $100 \%$ [11]. Moreover, the diagnosis is not clear and certain, because clinical and radiological symptoms of PCP are non-specific. Therefore, rapid and specific laboratory tests are necessary for early diagnosis of PCP [14]. The development of the PCR technique provided a new, reliable diagnostic method. As the PCR assay is about 100 times more sensitive than microscopy, the specificity of the PCR detection of $P$. jirovecii DNA in proper samples should be $100 \%$ [8]. Initially, the PCR technique was successfully used for amplification of $P$. jirovecii DNA from BAL specimens, achieving a sensitivity of more than $95 \%$ [16]. The sensitivity of PCR-based detection in non-invasive samples is certainly lower, not exceeding 70-80\% in HIV-positive patients.

Respiratory samples, such as BS, BAL, or ETA specimens are the material of choice for the diagnosis of PCP. 
Due to the high incidence of PCP in certain groups of immunocompetent and immunocompromised patients, besides cancer patients and transplant recipients, consideration of PCP prophylaxis is required in these groups as well.

The authors declare no conflict of interest.

\section{References}

1. Sing A, Trebesius K, Roggenkamp A, et al. (2000): Evaluation of diagnostic value and epidemiological implications of PCR for Pneumocystis carinii in different immunosuppressed and immunocompetent patient groups. J Clin Microbiol 38: 1461-1467.

2. Jarboui MA, Sellami A, Sellami H, et al. (2010): Molecular diagnosis of Pneumocystis jirovecii pneumonia in immunocompromised patients. Mycoses 53: 329-333.

3. Alanio A, Desoubeaux G, Sarfati C, et al. (2011): Real-time PCR assay-based strategy for differentiation between active Pneumocystis jirovecii pneumonia and colonization in immunocompromised patients. Clin Microbiol Infect 17: 1531-1537.

4. Samuel CM, Whitelaw A, Corcoran C, et al. (2011): Improved detection of Pneumocystis jirovecii in upper and lower respiratory tract specimens from children with suspected pneumocystis pneumonia using real-time PCR: a prospective study. BMC Infect Dis 11: 329-330.

5. Huggett JF, Taylor MS, Kocjan G, et al. (2008): Development and evaluation of a real-time PCR assay for detection of Pneumocystis jirovecii DNA in bronchoalveolar lavage fluid of HIV-infected patients. Thorax 63: 154-159.

6. Oren I, Hardak E, Finkelstein R, et al. (2011): Polymerase chain reaction-based detection of Pneumocystis jirovecii in bronchoalveolar lavage fluid for the diagnosis of pneumocystis pneumonia. Am J Med Sci 342: 182-185.

7. Flori P, Bellete B, Durand F, et al. (2004): Comparison between real-time PCR, conventional PCR and different staining techniques for diagnosing Pneumocystis jiroveci pneumonia from bronchoalveolar lavage specimens. J Med Microbiol 53: 603-607.

8. Durand-Joly I, Chabé M, Soula F, et al. (2005): Molecular diagnosis of Pneumocystis pneumonia. FEMS Immunol Med Microbiol 45: 405-410.

9. Larsen HH, Masur H, Kovacs JA, et al. (2002): Development and evaluation of a quantitative, touch-down, real-time PCR assay for diagnosing Pneumocystis carinii pneumonia. J Clin Microbiol 40: 490-494.

10. Gupta R, Iyer VK, Mirdha BR, et al. (2010): Role of cytology and polymerase chain reaction based detection of Pneumocystis jirovecii infection in bronchoalveolar lavage fluid. Acta Cytol 54: 296-302.

11. Pinlaor S, Mootsikapun P, Pinlaor P, et al. (2004): PCR diagnosis of Pneumocystis carinii on sputum and bronchoalveolar lavage samples in immuno-compromised patients. Parasitol Res 94: 213-218.

12. Huang L, Crothers K, Atzori C, et al. (2004): Dihydropteroate synthase gene mutations in Pneumocystis and sulfa resistance. Emerg Infect Dis 10: 1721-1728.

13. Rabodonirina M, Cotte L, Boibieux A, et al. (1999): Detection of Pneumocystis carinii DNA in blood specimens from human immunodeficiency virus-infected patients by nested PCR. J Clin Microbiol 37: 127-131.
14. Samuel CM, Whitelaw A, Corcoran C, et al. (2011): Improved detection of Pneumocystis jirovecii in upper and lower respiratory tract specimens from children with suspected pneumocystis pneumonia using real-time PCR: a prospective study. BMC Infect Dis 11: 329-331.

15. Worth LJ, Dooley MJ, Seymour JF, et al. (2005): An analysis of the utilisation of chemoprophylaxis against Pneumocystis jirovecii pneumonia in patients with malignancy receiving corticosteroid therapy at a cancer hospital. Br J Cancer 92: $867-872$.

16. Tamburrini E, Mencarini P, Visconti E, et al. (1998): Potential impact of Pneumocystis genetic diversity on the molecular detection of the parasite in human host. FEMS Immunol Med Microbiol 22: 37-49. 